\title{
Input Tracking and Output Fusion for Linear Systems
}

\author{
Xiaoming $\mathrm{Hu}^{1} \quad$ Ulf T. Jönsson ${ }^{1} \quad$ Clyde F. Martin ${ }^{2}$ \\ Dedicated to Prof Anders Lindquist, on the occasion of his 60th birthday
}

\footnotetext{
${ }^{1}$ Division of Optimization and Systems Theory, Royal Institute of Technology, 10044 Stockholm, Sweden

${ }^{2}$ Department of Mathematics and Statistics, Texas Tech University, Lubbock, Texas, USA
} 


\title{
Chapter 1
}

\section{Input Tracking and Output Fusion for Linear Systems}

\begin{abstract}
In this paper, the input-output behavior of a linear stable system is studied from a geometric point of view. Based on these results, it is discussed how to choose an output and how to fuse a number of outputs, to best track the input in stationarity.
\end{abstract}

\section{$1.1 \quad$ Introduction}

In this paper we first review in some detail how the input of a linear stable system is tracked by the output. Our motivation to further study this classical problem lies in that the results we obtain can be applied to other important problems such as optimal input tracking and sensor fusion. It is natural that before we can develop a procedure to choose an output or a combination of outputs (sensors) that best tracks an input (in the case that the input is at least partially unknown), we need to understand when a given output tracks the input.

In the second part of the paper, we will discuss a sensor fusion problem. There has been a vast literature on sensor fusion, see for example, the papers in [1] and the references therein. However, treatment of the problem from the input tracking point of view has to our knowledge not been addressed.

This paper is organized as follows. In section 2, we discuss the problem of how a given output tracks an input in stationarity. In sections 3 and 4 , we discuss the problem of how to choose an output, or a combination of sensors, 
to optimally track an input in stationarity. Finally, we use an example to illustrate our methods.

\subsection{Autonomous Linear Systems}

In this section we review some classical results on asymptotic input tracking. Consider a stable, controllable and observable SISO linear system:

$$
\begin{aligned}
\dot{x} & =A x+b u \\
y & =c x
\end{aligned}
$$

where $x \in R^{n}$ and $\sigma(A) \in C^{-}$.

We will consider the case when the input $u$ is generated by the following exogenous system:

$$
\begin{aligned}
\dot{w} & =\Gamma w \\
u & =q w
\end{aligned}
$$

where $w \in R^{m}$ and $\sigma(\Gamma) \in \bar{C}^{+}$. This exo-system can generally have a block diagonal Jordan realization

$$
\begin{aligned}
q & =\left(\begin{array}{ccc}
q_{1} & q_{2} & \ldots q_{M}
\end{array}\right) \\
\Gamma & =\operatorname{diag}\left(\Gamma_{1}, \Gamma_{2}, \ldots, \Gamma_{\mathrm{M}}\right)
\end{aligned}
$$

where each $q_{m}=\left(\begin{array}{lll}1 & 0 \ldots 0\end{array}\right)$ is a first unit vector of length $\operatorname{dim}\left(\Gamma_{m}\right)$ and each Jordan block corresponds either to polynomial, exponential, or sinusoidal functions. The output of the exo-system becomes

$$
u(t)=\sum_{m=1}^{M} q_{m} e^{\Gamma_{m} t} w_{0_{m}} .
$$

Such exo-systems can generate, for example, step functions, ramp functions, polynomials, exponentials, sinusoidals, and combinations of such functions.

Proposition 1.2.1 Suppose $A$ is a stable matrix, then all trajectories of $(x(t), w(t))$ tend asymptotically to the invariant subspace $S:=\{(x, w): x=\Pi w\}$, where $\Pi$ is the solution of

$$
A \Pi-\Pi \Gamma=-b q .
$$

On the invariant subspace, we have

$$
y(t)=c \Pi w(t) .
$$


The proof of this proposition can be found, for example, in [5], and there is also a vast literature on the nonlinear case $[3,6]$.

Using the matrix $\Pi$ we have that the output of the linear system in the steady-state can be represented as

$$
y=c \Pi w .
$$

Proposition 1.2.2 Let the system

$$
\dot{w}=\Gamma w, \quad u=q w
$$

be observable and no eigenvalue of $\Gamma$ is a transmission zero of (1.1). Then the system on the invariant subspace

$$
\dot{w}=\Gamma w, \quad y=c \Pi w
$$

is also observable.

Proof: A similar proof can be found in [5]. Since this result will be used several times later on, we give a full proof here.

We first need to establish that under the hypotheses, the composite system

$$
\begin{aligned}
\left(\begin{array}{c}
\dot{x} \\
\dot{w}
\end{array}\right) & =\left(\begin{array}{cc}
A & b q \\
0 & \Gamma
\end{array}\right)\left(\begin{array}{c}
x \\
w
\end{array}\right) \\
y & =c x
\end{aligned}
$$

is observable. Methods for proving similar results can be found, for example, in $[2]$.

Define

$$
H(s)=\left(\begin{array}{cc}
s I-A & -b q \\
0 & s I-\Gamma \\
c & 0
\end{array}\right) .
$$

By Hautus test we know that the system is observable if and only if

$$
\operatorname{rank}(H(s))=n+m \quad \forall s .
$$

If $s$ is not an eigenvalue of $\Gamma$, it is easy to see that $\operatorname{rank}(H(s))=n+m$ since $(c, A)$ is observable. Now suppose $s$ is an eigenvalue of $\Gamma$,

$$
H(s)=\left(\begin{array}{ccc}
s I-A & b & 0 \\
0 & 0 & I_{m} \\
c & 0 & 0
\end{array}\right)\left(\begin{array}{cc}
I_{n} & 0 \\
0 & -q \\
0 & s I-\Gamma
\end{array}\right) .
$$


If $s$ is not a transmission zero of (1.1), then and only then the first matrix on the right-hand side has rank $n+1+m$. The second has rank $n+m$ since $(q, \Gamma)$ is observable. By Sylvester's inequality, we have

$$
\operatorname{rank}(H(s)) \geq n+1+m+n+m-(n+m+1)=n+m .
$$

Therefore $\operatorname{rank}(H(s))=n+m$.

Now we do a coordinate change $\bar{x}=x-\Pi w$. Then (1.4) becomes

$$
\begin{aligned}
\left(\begin{array}{c}
\dot{\bar{x}} \\
\dot{w}
\end{array}\right) & =\left(\begin{array}{cc}
A & 0 \\
0 & \Gamma
\end{array}\right)\left(\begin{array}{c}
\bar{x} \\
w
\end{array}\right) \\
y & =c \bar{x}+c \Pi w
\end{aligned}
$$

It is straight forward to see that

$$
\left((c, c \Pi),\left(\begin{array}{cc}
A & 0 \\
0 & \Gamma
\end{array}\right)\right)
$$

is observable implies $(c \Pi, \Gamma)$ is so too. Q.E.D.

\section{An observer for the input}

Based on this result it follows that the input $u$ can be reconstructed by the observer

$$
\begin{aligned}
\dot{\hat{w}} & =\Gamma \hat{w}+L(y-c \Pi \hat{w}) \\
\hat{u} & =q \hat{w}
\end{aligned}
$$

where the vector $L$ can be designed such that the eigenvalues of $\Gamma-L c \Pi$ can be placed anywhere we desire in the complex plane. This is a reduced order observer since the dynamics corresponding to $A$ is not included in the observer equation. One of the prices we pay for this is that the convergence of the observer is restricted by the transients corresponding to the eigenvalues of $A$. Indeed, if $\bar{x}=x-\Pi w, \bar{w}=w-\hat{w}$, and $\bar{u}=q(w-\hat{w})$ then the error dynamics becomes

$$
\begin{aligned}
\left(\begin{array}{c}
\dot{\bar{x}} \\
\dot{\bar{w}}
\end{array}\right) & =\left(\begin{array}{cc}
A & 0 \\
-L c & \Gamma-L c \Pi
\end{array}\right)\left(\begin{array}{l}
\bar{x} \\
\bar{w}
\end{array}\right) \\
\bar{u} & =q \bar{w}
\end{aligned}
$$

We will in the next section see that under special conditions it is possible to design the output such that the input is reconstructed in stationarity. This will give a memoryless observer, which is preferable from a computational point of view. 


\subsection{Exact Input Tracking}

We will now discuss how Proposition 1.2.1 can be used to determine an appropriate output in order to track the input exactly in stationarity. It follows obviously that the output tracks the input if the vector $c$ is chosen such that

$$
(c \Pi-q) e^{\Gamma t} w_{0}=0
$$

where $w_{0}$ is the initial state of (1.2) that generates the input. This is clearly the case if $c \Pi=q$. If $\Pi$, for example, has full column rank, then it is possible to design an output $c$ for perfect input tracking in stationarity. We will show below that this is the case if $(A, b)$ is controllable, $(q, \Gamma)$ is observable and $\operatorname{dim}(A) \geq \operatorname{dim}(\Gamma)$.

In some way one may view this problem as a dual one to the output regulation problem discussed in [4]. In this section, we discuss some necessary and sufficient conditions.

Theorem 1.3.1 Suppose $(q, \Gamma)$ is observable and $(A, b)$ controllable. Then a necessary and sufficient condition for the existence of a $c$, such that $c \Pi=q$, is that the dimension of $A$ is greater or equal to that of $\Gamma$.

Proof: We can rewrite $\dot{x}=A x+b u$ in the canonical form:

$$
\begin{aligned}
\dot{x}_{1} & =x_{2} \\
& \vdots \\
\dot{x}_{n-1} & =x_{n} \\
\dot{x}_{n} & =-\sum_{i=1}^{n} a_{i} x_{i}+k u,
\end{aligned}
$$

where $k \neq 0$ and $\rho(s)=s^{n}+\sum_{i=1}^{n} a_{i} s^{i-1}$ is Hurwitz. In the steady state, by Proposition 1.2.1 we have

$$
x_{1}=\pi_{1} w,
$$

where $\pi_{1}$ is the first row of $\Pi$. Since $x_{i}=\pi_{1} \Gamma^{i-1} w$, for $i=1, \ldots, n$, we have

$$
\Pi=\left(\begin{array}{c}
\pi_{1} \\
\pi_{1} \Gamma \\
\vdots \\
\pi_{1} \Gamma^{n-1}
\end{array}\right) .
$$


Thus,

$$
\pi_{1} \Gamma^{n}=-\sum_{i=1}^{n} a_{i} \pi_{1} \Gamma^{i-1}+k q .
$$

Since by assumption $\Gamma$ does not have any eigenvalue in the open left-half plane, we have

$$
\pi_{1}=k q \rho(\Gamma)^{-1}
$$

If there exists a $c$, such that

$$
q=c \Pi=\sum_{i=1}^{n} c_{i} \pi_{1} \Gamma^{i-1}
$$

then

$$
q=k q \rho(\Gamma)^{-1} \sum_{i=1}^{n} c_{i} \Gamma^{i-1},
$$

or,

$$
q\left(I-k \rho(\Gamma)^{-1} \sum_{i=1}^{n} c_{i} \Gamma^{i-1}\right)=0 .
$$

Denote $\Delta=I-k \rho(\Gamma)^{-1} \sum_{i=1}^{n} c_{i} \Gamma^{i-1}$. It is easy to show that

$$
\Delta=\left(\Gamma^{n}+\sum_{i=1}^{n}\left(a_{i}-k c_{i}\right) \Gamma^{i-1}\right) \rho(\Gamma)^{-1}
$$

thus $q \Delta=0$ if and only if

$$
q \Gamma^{n}+\sum_{i=1}^{n}\left(a_{i}-k c_{i}\right) q \Gamma^{i-1}=0 .
$$

Since $(q, \Gamma)$ is observable, $(1.10)$ has a solution if and only if $n$ is greater or equal to the dimension of $\Gamma$. Q.E.D.

Corollary 1.3.1 If $\operatorname{dim}(A) \geq \operatorname{dim}(\Gamma)$, then $\Pi$ has full column rank, and thus there exists a $c$, such that,

$$
c \Pi=q .
$$

Moreover, if $\operatorname{dim}(A)=\operatorname{dim}(\Gamma)$, then such $c$ is unique. 
Proof: It follows from (1.8), (1.9), (1.10), and observability of the exo-system (1.2). Indeed,

$$
\Pi=\left(\begin{array}{c}
\pi_{1} \\
\pi_{1} \Gamma \\
\vdots \\
\pi_{1} \Gamma^{n-1}
\end{array}\right)=k\left(\begin{array}{c}
q \\
q \Gamma \\
\vdots \\
q \Gamma^{n-1}
\end{array}\right) \rho(\Gamma)^{-1}
$$

which has full rank since $(q, \Gamma)$ is observable.

Q.E.D.

Corollary 1.3.2 Suppose $\operatorname{dim}(A)=n \geq \operatorname{dim}(\Gamma)=m$, then there exists a $c$ such that $c \Pi=q$ and the resulting system (1.4) is observable and (1.1) does not have any transmission zero that is also an eigenvalue of $\Gamma$.

Proof: Consider the canonical form (1.7). Suppose the characteristic polynomial for $\Gamma$ is $\rho_{\Gamma}(s)=s^{m}+\sum_{i=1}^{m} \gamma_{i} s^{i-1}$. It follows from (1.10) and CayleyHamilton that

$$
c_{i}=\frac{1}{k}\left(a_{i}-\bar{\gamma}_{i}\right) \quad i=1, \ldots, n,
$$

where $\bar{\gamma}_{i}=0 \forall i<n-m+1$ and $\bar{\gamma}_{i}=\gamma_{i-n+m}$ otherwise, is a solution such that $c \Pi=q$. It then follows from the fact that $A$ and $\Gamma$ do not share any eigenvalue, that no eigenvalue $s_{0}$ of $A$ or $\Gamma$ is a root of

$$
\sum_{i=1}^{n} c_{i} s_{0}^{i-1}=\frac{1}{k} \sum_{i=1}^{n} a_{i} s_{0}^{i-1}-\frac{s_{0}^{n-m}}{k} \sum_{i=1}^{m} \gamma_{i} s_{0}^{i-1} .
$$

Indeed, if $s_{0}$ is for example a root of the characteristic polynomial of $A$, the above expression reduces to

$$
-\frac{s_{0}^{n-m}}{k} \rho_{\Gamma}\left(s_{0}\right)
$$

which must be nonzero. Thus, no transmission zero of the corresponding (1.1) is an eigenvalue of $\Gamma$ and the pair $(c, A)$ is observable. From the proof of Proposition 1.2.2 we derive that (1.4) is observable.

Q.E.D.

We have shown under the assumptions of Corollary 1.3.1 that the input $u$ can be reconstructed simply as

$$
\hat{u}=c x,
$$

where, e.g. $c=q \Pi^{\dagger}$, where $\Pi^{\dagger}$ is the pseudo inverse. The tracking error satisfies (where $\bar{x}=x-\Pi w$ )

$$
\bar{u}=u-\hat{u}=c x-q w=c \bar{x}+c \Pi w-q w=c \bar{x}
$$


Hence, the error dynamics in this case becomes

$$
\begin{aligned}
& \dot{\bar{x}}=A \bar{x} \\
& \bar{u}=c \bar{x}
\end{aligned}
$$

which, as for the observer in (1.5), has its rate of convergence limited only by the eigenvalues of $A$.

The exo-system will in many applications have significantly larger dimension than the linear system (1.1) and then there only could exist a solution to (1.6) for special choices of initial condition $w_{0}$ of the exo-system. In the next section we discuss a strategy for fusing the output of a number of outputs in order to minimize the steady state tracking error.

\subsection{Output Fusion for Input Tracking}

We will here consider a special sensor fusion problem where we try to minimize the tracking error by appropriately combining the outputs of a number of sensors. A sensor in our terminology means a particular choice of $c_{k}$ matrix. If the state space model represents physical variables, then typically each $c_{k}$ corresponds to one state variable. The idea is that the sensor fusion block

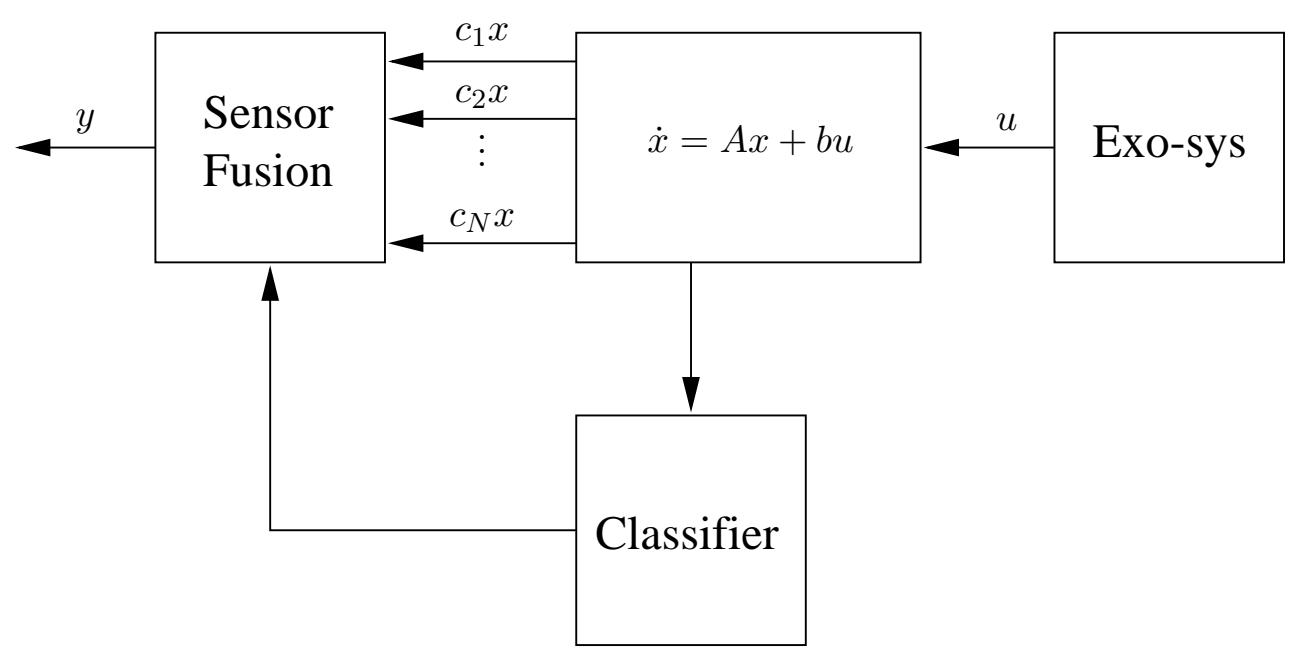

Figure 1.1: Sensor fusion set-up.

should determine a linear combination of the sensor signals such that the 
output

$$
y=\sum_{k=1}^{N} \alpha_{k} c_{k} x
$$

tracks the input $u$ in stationarity. We will here discuss how this sensor fusion idea works for the case when the input is generated by an observable exosystem of the form (1.2).

\section{The Classifier Block}

We first need a classifier block in order to determine what Jordan blocks are active in the generation of the input $u$, i.e. it determines a set $\mathcal{M} \subset\{1, \ldots, M\}$ of indices such that the input can be represented as

$$
u(t)=\sum_{m \in \mathcal{M}} q_{m} e^{\Gamma_{m} t} w_{0_{m}}
$$

We will see below that this information sometimes is enough to obtain perfect tracking. However, it is generally important to use as much information on the vector $w_{0}$ as possible in order to obtain better tracking. For example, if we in addition to $\mathcal{M}$ also obtain an estimate $\hat{w}_{0_{\mathcal{M}}}=\left[\hat{w}_{0_{m_{1}}}, \ldots, \hat{w}_{0_{m_{n}}}\right]$ of the initial condition then our ability to reconstruct the input improves. Even qualitative information such as the relative amplitude of the various blocks is useful.

\section{The Sensor Fusion Block}

This block takes as input the classification $\mathcal{M}$ and maps it to a vector $\alpha$ that minimizes the steady state tracking error for the output (1.11) according to some cost criterion. We will discuss this in more detail below where we also give necessary and sufficient conditions for obtaining perfect tracking. In more sophisticated schemes we may also use an estimate $\hat{w}_{0_{\mathcal{M}}}$ of the initial condition of the exo-system. This may give better tracking, however at the price of more complex classifier and sensor fusion blocks. Note that this scheme will be independent of the state space realization and the convergence to the steady state solution depends on the spectrum of $A$.

A main practical motivation for our sensor fusion scheme is due to the limited communication and computation resources in many embedded systems, such as mobile robotic systems. There is a need to develop "cheap" sensing algorithms. The central idea in our scheme is to optimally combine the existing sensors for state variables to measure the external signals. This optimization 
can be done off-line and then the sensor fusion block only needs to use a table look-up to decide the parameter vector $\alpha$. The only remaining issue is how to design the online classifier.

The most natural way from a systems point of view is perhaps to use a dynamical observer (in discrete time) to identify qualitatively the initial condition (or the active $\Gamma$ blocks) and then shut it down. This is possible since the state of the exo-system is observable from the output from any sensor that satisfies the conditions of Proposition 1.2.2. However, this approach could be computationally expensive even if we only run it once in a while. A more refined scheme is discussed for a special case below.

For many practical systems, it is perhaps more realistic to design the classifier based on other sensors that sense the interaction of the system with the environment (such as laser scanners and video cameras), or/and on the nature of application the system is operated for. In this way, typically only a range of the $\Gamma$ blocks (such as a frequency range) can be identified.

\section{An Example Classifier}

Let us consider the case when only one Jordan block of $\Gamma$ is active at a time. Then we can construct a classifier consisting of a bank of discrete time observers. Each observer in the classifier corresponds to a particular Jordan block. The idea is to initially activate all observers in order to decide which block is active. Once this is done, all observers are shut down except the one corresponding to the active Jordan block. This observer is used to detect changes in the exo-system. By running the monitor on low sampling frequency a minimum computational effort is spent in the classifier.

Let us discuss how to construct such an observer for block $\Gamma_{m}$. Zero order hold sampling of the dynamics gives

$$
\left(\begin{array}{l}
x_{k+1} \\
w_{k+1}
\end{array}\right)=\left(\begin{array}{cc}
A_{d} & b_{m, d} q_{m, d} \\
0 & \Gamma_{m, d}
\end{array}\right)\left(\begin{array}{l}
x_{k} \\
w_{k}
\end{array}\right)
$$

where $h$ is the sampling time, $x_{k}=x(k h), w_{k}=w(k h), A_{d}=e^{A h}, \Gamma_{m, d}=$ $e^{\Gamma_{m} h}$, and $b_{m, d}, q_{m, d}$ are matrices of full rank such that

$$
b_{m, d} q_{m, d}=\int_{0}^{h} e^{A(h-s)} b q e^{\Gamma_{m} s} d s .
$$

We can use exactly the same arguments as in the proof of Proposition 1.2.1 to show that the functions $\left(x_{k}, w_{k}\right)$ converges to an invariant subspace $S_{m}=$ $\left\{(x, w): x=\Pi_{m} w\right\}$. It should be intuitively clear that the invariant subspace 
is the same as in continuous time. The following proposition gives a formal mathematical proof.

Proposition 1.4.1 The sampled functions converge to the invariant subspace $S_{m}=\left\{(x, w): x=\Pi_{m} w\right\}$, where

$$
A \Pi_{m}-\Pi_{m} \Gamma_{m}=b q_{m}
$$

Proof: Let $u_{k}=q w_{k}$ and assume $x_{k}=\Pi_{m} w_{k}$. It is straight forward to derive that $x_{k}=\Pi_{m} w_{k}$ is invariant if and only if

$$
A_{d} \Pi_{m}-\Pi_{m} \Gamma_{m}=b_{m, d} q_{m, d} .
$$

This can be written

$$
\begin{gathered}
e^{A h} \Pi_{m}-\Pi_{m} e^{\Gamma_{m} h}=-\int_{0}^{h} e^{A(h-s)} b q_{m} e^{\Gamma_{m} s} d s \\
\Leftrightarrow \quad e^{A h} \Pi_{m} e^{-\Gamma_{m} h}-\Pi_{m}=-\int_{0}^{h} e^{A(h-s)} b q_{m} e^{-\Gamma_{m}(h-s)} d s
\end{gathered}
$$

This is a discrete time Lyapunov equation so we get

$$
\begin{aligned}
\Pi_{m} & =\sum_{i=0}^{\infty} e^{A i h} \int_{0}^{h} e^{A(h-s)} b q_{m} e^{-\Gamma_{m}(h-s)} d s e^{-\Gamma_{m} i h} \\
& =\sum_{i=0}^{\infty} \int_{i h}^{(i+1) h} e^{A \tau} b q_{m} e^{-\Gamma_{m} \tau} d \tau=\int_{0}^{\infty} e^{A \tau} b q_{m} e^{-\Gamma_{m} \tau} d \tau
\end{aligned}
$$

which is the solution of the Lyapunov equation $A \Pi_{m}-\Pi_{m} \Gamma_{m}=b q_{m}$. The convergence to the subspace can be proven similarly to that in the proof of Proposition 1.2.1.

Q.E.D.

We will next consider observability of the pairs $\left(c \Pi_{m}, \Gamma_{m d}\right)$ on the invariant subspace $S_{m}=\left\{(x, w): x=\Pi_{m} w\right\}$. It is well known that the sampled system $\left(c \Pi_{m}, \Gamma_{m d}\right)$ is observable if and only if the continuous time system $\left(c \Pi_{m}, \Gamma_{m}\right)$ is observable. Hence, from Proposition 1.2.2 we know that a sufficient condition is that the exo-system $\left(q_{m}, \Gamma_{m}\right)$ is observable and that no eigenvalue of $\Gamma_{m}$ is a transmission zero of the system (1.1). The next proposition shows that it is possible to design one sensor that works for all Jordan blocks.

Proposition 1.4.2 Suppose all pairs $\left(q_{m}, \Gamma_{m}\right)$ are observable. Then there exists a sensor combination, $c$, such that all pairs $\left(c \Pi_{m d}, \Gamma_{m d}\right)$ are observable on the corresponding invariant subspace. 
Proof: From the above discussion and Proposition 1.2.2 it follows that it is enough to find $c$ such that $(c, A)$ is observable and (1.1) does not have any transmission zero at the eigenvalues of the $\Gamma_{m}$. Such a $c$ is always possible to design since only a finite number of constraints are imposed.

Assume now that we have sensors $c_{o}$, which corresponds to the output $z$, such that the pairs $\left(c_{o} \Pi_{m}, \Gamma_{m d}\right)$ are observable. We can then use the following block of observers

$$
\hat{w}_{k+1}=\Gamma_{m, d} \hat{w}_{k}+L_{m}\left(z_{k}-c_{o} \Pi_{m} \hat{w}_{k}\right)
$$

where the observer gains $L_{m}$ are designed such that the eigenvalues of $\Gamma_{m, d}-$ $L_{m} c_{o} \Pi_{m}$ are stable. The observer that corresponds to the active Jordan block has error dynamics

$$
\left(\begin{array}{c}
\bar{x}_{k+1} \\
\bar{w}_{k+1}
\end{array}\right)=\left(\begin{array}{cc}
A_{d} & 0 \\
-L_{m} c_{o} & \Gamma_{m, d}+L_{m} c_{o} \Pi_{m}
\end{array}\right)\left(\begin{array}{l}
\bar{x}_{k} \\
\bar{w}_{k}
\end{array}\right)
$$

which convergences to zero. By proper design of $L_{m}$ the convergence rate is determined by the eigenvalues of $A_{d}$. All other observers have the error dynamics

$$
\bar{w}_{k+1}=\left(\Gamma_{m, d}-L_{m} c_{o} \Pi_{m}\right) \bar{w}_{k}+\left(\Gamma_{n, d}-\Gamma_{m, d}-L_{m} c_{o}\left(\Pi_{n}-\Pi_{m}\right)\right) w_{k}
$$

The matrix $\left.\Lambda=\Gamma_{n, d}-\Gamma_{m, d}-L_{m} c_{o}\left(\Pi_{n}-\Pi_{m}\right)\right)$ can without loss of generality be assumed to be nonzero, which implies that $\bar{w}_{k}$ does not converge to zero. To see that $\Lambda$ generically is nonzero, let us suppose $\Lambda=0$. This implies

$$
\left(\Gamma_{m, d}-L_{m} c_{o} \Pi_{m}\right) e^{-\Gamma_{n} h}-I=-L_{m} c_{o} \Pi_{n} e^{-\Gamma_{n} h}
$$

On the other hand,

$$
\left(\Gamma_{m, d}-L_{m} c_{o} \Pi_{m}\right) \Psi e^{-\Gamma_{n} h}-\Psi=-L_{m} c_{o} \Pi_{n} e^{-\Gamma_{n} h}
$$

has the unique solution

$$
\Psi=\sum_{i=0}^{\infty}\left(\Gamma_{m, d}-L_{m} c_{o} \Pi_{m}\right)^{i} L_{m} c_{o} \Pi_{n} e^{-\Gamma_{n} h(i+1)} .
$$

Generically, we have $\Psi \neq I$, thus we can design $L_{m}$ such that $\Lambda \neq 0$.

In the decision logic block we simply need to compare the magnitude of the error signals $\bar{w}_{m}$ from observers. Under our hypothesis that only one Jordan block at a time is active it follows that only one error signal will converge to zero. 
Once it is decided which Jordan block is active the classifier goes into the monitoring mode, where the observer corresponding to the active Jordan block is used to detect a change in the input signals. The sampling frequency in the monitoring mode can often be chosen significantly lower than in the detection mode. Suppose, for example, that the Jordan blocks correspond to sinusoidals with distinct frequencies. Then by choosing the sampling frequency such that these frequencies fold into distinct locations, we can detect a change of frequency with a significantly lower sampling rate than the Nyquist frequency.

\section{Perfect Steady-state Tracking}

Assume we are given $K$ sensor combinations $c_{1}, \ldots, c_{K}$. We derive a sufficient (and in a sense also necessary) condition for obtaining perfect steady state tracking using these sensors. We have the following result.

Proposition 1.4.3 Suppose $\mathcal{M}=\left\{m_{1}, \ldots, m_{n}\right\} \subset\{1, \ldots, M\}$ are the active Jordan blocks. Then we can obtain perfect tracking if

$$
q_{\mathcal{M}}^{T} \in \operatorname{Im}\left(\Pi_{\mathcal{M}}^{T} C^{T}\right)
$$

where $A \Pi_{\mathcal{M}}-\Pi_{\mathcal{M}} \Gamma_{\mathcal{M}}=-b q_{\mathcal{M}}$ and

$$
\begin{aligned}
\Gamma_{\mathcal{M}} & =\operatorname{diag}\left(\Gamma_{m_{1}}, \ldots, \Gamma_{m_{n}}\right) \\
q_{\mathcal{M}} & =\left(q_{m_{1}}, \ldots, q_{m_{n}}\right) \\
C^{T} & =\left[\begin{array}{lll}
c_{1}^{T} & \ldots & c_{K}^{T}
\end{array}\right]
\end{aligned}
$$

Proof: The steady state output will be $y=\alpha C \Pi_{\mathcal{M}} w_{\mathcal{M}}(t)$, where $w_{\mathcal{M}}(t)=$ $e^{\Gamma_{\mathcal{M}} t} w_{0_{\mathcal{M}}}$. Hence, we obtain perfect tracking since our assumption implies that there exists a solution to $\alpha C \Pi_{\mathcal{M}}=q_{\mathcal{M}}$. Note that this condition is necessary if $w_{0_{\mathcal{M}}}$ is allowed to take any value. Q.E.D.

If the condition of the proposition holds then we normally want to find the vector $\alpha$ with the minimum nonzero coefficients such that

$$
\alpha C \Pi_{\mathcal{M}}=q_{\mathcal{M}}
$$

in order to minimize the number of sensors used. This can be done off-line and then the sensor fusion block only need to use a table look-up to decide the vector $\alpha$. 


\section{Approximate Tracking}

It will often happen that we have too few sensors or too poor knowledge of the exo-system to obtain perfect tracking. In such cases we need to optimize the sensor fusion in order to get best tracking in some average sense.

Suppose $\Gamma(\delta), q(\delta)$ is an uncertain parameterization of the exo-system, where $\delta \subset \Delta$ is the uncertain parameters. We let $\Delta=\{0\}$ correspond to the case when we have perfect knowledge of the exo-system. If $\Delta \neq\{0\}$ or if the condition in Proposition 1.4.3 does not hold then we let the sensor fusion be determined by the solution to some optimization problem

$$
\min _{\alpha} \mathcal{L}_{\Delta}(|\alpha C \Pi(\delta)-q(\delta)|)
$$

where $\mathcal{L}_{\Delta}$ is some functional over $\Delta$ and $\Pi(\delta)$ is the solution to $A \Pi(\Gamma(\delta))-$ $\Pi(\delta) \Gamma(\delta)=-b q(\delta)$. Some examples are given in [5].

\section{An Example of optimal output design}

As an example to demonstrate our methodology, we consider a car-like base and a robot arm mounted on it. By using the homogeneous representation of rigid body motions, we can easily compute the position of the end-effector, relative to the base, $r_{A}^{B}$, and thus the kinematic model as

$$
\dot{x}_{A}=f\left(x_{A}, u\right) .
$$

Under the assumptions that the velocity of the car is constant, and that the side slip angle is small, we can get a simplified model of the base vehicle as follows:

$$
\begin{aligned}
& \dot{x}=v \cos _{B}(\psi+\beta) \\
& \dot{y}=v \sin _{B}(\psi+\beta) \\
& \dot{\beta}+r=\frac{f_{f}+f_{r}}{m v}=a_{11} \beta+a_{12} r+b_{11} \delta_{f} \\
& \dot{\psi}=r \\
& \dot{r}=\frac{f_{f} l_{f}-f_{r} l_{r}}{J}=a_{21} \beta+a_{22} r+b_{21} \delta_{f}+d(t),
\end{aligned}
$$

where $(x, y)$ is the center of gravity of the vehicle, $m$ is the vehicle mass and $J$ is the moment of inertia, and the $a$ and $b$ coefficients depend on the physical parameters of the vehicle.

The disturbance $d(t)$ could be for example, side wind or roughness on the road surface. 
This example shows that by measuring the orientation $(\psi)$ and yaw rate $(r)$, it is possible to recover $d(t)$ in some cases, which shall be useful to know for the control of the arm.

The advantage of using the output fusion method, in comparison to the observer method, is that the lateral dynamics of the car is typically very fast. Thus this method converges fast, while not having the transient peakings that might be induced by the pole placements of an observer.

Figure 1.2 shows a simulation where by combining $r(t)$ and $\psi(t)$ we can track a sinusoidal disturbance $d(t)$ in stationarity. The upper diagram shows the convergence of the output and the lower bound shows the error $y-u$.
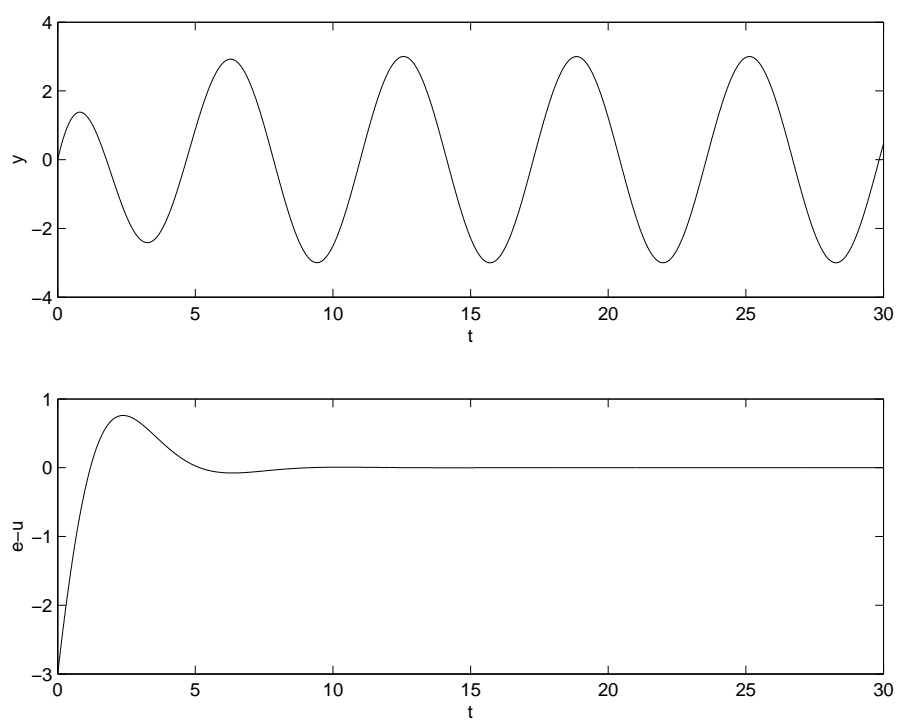

Figure 1.2: Simulation of the sensor fusion.

\subsection{Concluding Remarks}

We have discussed conditions for steady state input tracking for stable linear systems. It has been shown that these results can be used to devise a sensor fusion scheme for input tracking in some situations. We need to further study the robustness issue of such methods (for example, when there are uncertainties in $\Gamma$ and/or in the measurements) and ways to generalize them. 
18CHAPTER 1. INPUT TRACKING AND OUTPUT FUSION FOR LINEAR SYSTEMS 


\section{Bibliography}

[1] Proceedings of the IEEE, volume 85, Jan 1997. Special issue on sensor fusion.

[2] C.T. Chen. Linear system theory and design. HRW, New York, 1984.

[3] E. Coddington and N. Levinson. Theory of ordinary differential equations. McGraw-Hill, New York, 1984.

[4] B. Francis. The linear multivariable regulator problem. SIAM J. Control. Optim., 15:486-505, 1977.

[5] X. Hu, U. Jönsson, and C. Martin. Input tracking for stable linear systems. In Proceedings of the 2002 IFAC Congress, Barcelona, July 2002.

[6] A. Kelly. The stable, center-stable, center, center-unstable, and unstable manifolds. J.Diff.Eqns, 3:546-570, 1967. 